\title{
On the centipede genus Schizotergitius Verhoeff, 1930, with a redescription of Schizotergitius altajicus Loksa, 1978 and a key to the genera of the family Lithobiidae in Central Asia (Chilopoda: Lithobiomorpha)
}

\section{О костянках рода Schizotergitius Verhoeff, 1930, с переописанием Schizotergitius altajicus Loksa, 1978 и ключом для родов семейства Lithobiidae Центральной Азии (Chilopoda: Lithobiomorpha)}

\author{
Yu.V. Dyachkov ${ }^{1}$, G.Sh. Farzalieva ${ }^{2}$, L. Dányi ${ }^{3}$ \\ Ю.В. Аьячков ${ }^{1}$, Г.Ш. Фарзалиева ${ }^{2}, \Lambda$. Ааньи $^{3}$
}

\footnotetext{
${ }^{1}$ Altai State University, Lenin Avenue, 61, Barnaul 656049, Russia. E-mail: dyachkov793@mail.ru

Алтайский государственный университет, проспект Ленина, 61, Барнаул 656049, Россия.

2 Perm State University, Bukireva Street, 15, Perm 614600, Russia.

2 Пермский государственный национальный исследовательский университет, ул. Букирева, 15, Пермь 614990, Россия.

${ }^{3}$ Centre for Agricultural Research, Institute for Soil Sciences, Herman Ottó út 15, Budapest H-1022, Hungary.
}

KEY WORDS: Lithobiinae, Schizotergitius, taxonomy, faunistics, diagnosis, description, genera, key, Central Asia КЛЮЧЕВЫЕ СЛОВА: Lithobiinae, Schizotergitius, таксономия, фаунистика, диагноз, описание, ключ, Центральная Азия.

ABSTRACT. The genus Schizotergitius Verhoeff, 1930, subfamily Lithobiinae, is briefly redescribed, rediagnosed, and shown to comprise only two species: Schizotergitius longiventris Verhoeff, 1930 (the type species from Tajikistan) and S. altajicus Loksa, 1978 (Mongolia). Based on type and fresh material of both sexes, $S$. altajicus is redescribed, and its distribution is mapped. An identification key to all eight genera of Lithobiidae occurring in Central Asia is presented.

РЕЗЮМЕ. Для рода Schizotergitius Verhoeff, 1930 (подсемейство Lithobiinae) даны краткое переописание и новый диагноз. Показано, что род включает только два вида: Schizotergitius longiventris Verhoeff, 1930 (типовой вид из Таджикистана) и S. altajicus Loksa, 1978 (Монголия). Вид S. altajicus переописан по типовому и новому материалу обоих полов, и его распространение картировано. Дан ключ для определения всех восьми родов Lithobiidae, отмеченных в Центральной Азии.

\section{Introduction}

The genus Schizotergitius Verhoeff, 1930 is one of the most poorly studied and badly circumscribed genera of lithobiomorph centipedes. The type species of the genus,
S. longiventris Verhoeff, 1930, was described from Tajikistan [Verhoeff, 1930], based only on four $\sigma^{7}$ syntypes. Verhoeff specified elongated sternites and tergites, and each of tergites $3,5,8,10$ and 12 divided into two lobes as being the main characters to distinguish that genus.

Loksa [1978] added further two species to the genus, both from Mongolia: S. styliferus Loksa, 1978 and S. altajicus Loksa, 1978. The former species, based on the morphological characters of the females gonopods, was later transferred to Hessebius Verhoeff, 1941, being presently referred to as $H$. styliferus (Loksa, 1978) [Pei et al., 2010]. As a result, Schizotergitius has hitherto been considered as encompassing two species only: $S$. longiventris and S. altajicus.

While studying new material from western Mongolia [Dyachkov, 2017: 35; Farzalieva et al., 2017: 124], specimens of $S$. altajicus were also revealed. Their examination showed that Loksa [1978] had omitted some details of females gonopod structure, nor had he illustrated certain males secondary sexual characters.

The main objective of the present paper is to provide a detailed redescription of $S$. altajicus, to update the diagnosis of Schizotergitius, and to incorporate it into a key to all genera of lithobiid centipedes known to occur in Central Asia: Bothropolys Wood, 1862 (Ethopolyinae Chamberlin, 1915), Australobius Chamberlin, 1920, Dis-

How to cite this article: Dyachkov Yu.V., Farzalieva G.Sh., Dányi L. 2021. On the centipede genus Schizotergitius Verhoeff, 1930, with a redescription of Schizotergitius altajicus Loksa, 1978 and a key to the genera of the family Lithobiidae in Central Asia (Chilopoda: Lithobiomorpha) // Russian Entomol. J. Vol.30. No.3. P.361-371. doi: $10.15298 /$ rusentj.30.3.16 
phaerobius Attems, 1926, Hessebius Verhoeff, 1941, Lithobius Leach, 1814, Schizotergitius Verhoeff, 1930, and Validifemur Ma, Song et Zhu, 2007 (Lithobiinae Verhoeff, 1907) [Loksa, 1978; Zalesskaja, 1978; Eason, 1997; Ma et al., 2007, 2014; Farzalieva et al., 2017; Ganske et al., 2020].

\section{Materials and methods}

We have re-examined the paratypes of Schizotergitius altajicus Loksa, 1978, kept in the Hungarian Natural History Museum, Budapest (HNHM). Although the HNHM had been indicated as the repository for the holotype as well, it could not be relocated. We have also studied additional 39 specimens coming from different parts of western Mongolia (Fig. 1), all donated by A.A. Fomichev. This additional material is shared between the collections of the Zoological Museum of the Moscow State University, Moscow (ZMMU), the Perm State University, Perm (PSU), the Altai State University, Barnaul (ASU), and HNHM.

The counts of tergites and standardized terminology follow Bonato et al. [2010]. The term, "a posterior median notch", is adopted from Eason [1986a] and Zapparoli, Edgecombe [2011: 383], as well as "a pretarsal anteroventral spine", the term omitted by Bonato et al. [2010] and discussed as "an anteroventral spur" by Edgecombe [2004].

Measurements. The total body length is measured from the fore margin of the cephalic plate to the rear edge of the postpedal tergite. A small piece of a thin and light glass slide was used for stretching the specimen in a dorsoventral position for measuring the tergites. The length and width of the tergites were measured using FAST Software Version 1.0 [Vaganov et al., 2020].

Plectrotaxy. Legs spinulation data are presented in a tabular form. Variations in plectrotaxy between specimens are given in parentheses. The number of coxal pores on legs $12-15$ is given as a formula where a sequence of Arabic numerals means the number of pores from legs 12 to 15 .

Most of the pictures were taken using an Olympus DP74 digital camera attached to an Olympus SZX16 stereo microscope. SEM micrographs were prepared at the PSU applying a Hitachi TM3000 scanning electron microscope with a back-scatter electron detector. The line drawings were executed by one of us (GSF) using a Meiji EMZ5 stereo microscope. To closer examine the cephalic plate, forcipules, mandibles, maxillary complex and + gonopods, they were mounted in permanent slides utilizing euparal or sandarac medium [Krasheninnikov, 2011].

The distribution map was generated using SimpleMappr software [Shorthouse, 2010].

Locality data repeat the original labels, additional information is given in square brackets. The following abbreviations are used in the text and tables: collectors: $\mathrm{OB}$ - O. Berdaulet, VD - V.V. Doroshkin, AF A.A. Fomichev, AK - A.A. Kechaykin, DN - D. Nuralla, RY — R.V. Yakovlev; morphology: T/TT tergite(s), S/SS - sternite(s), Tim - intermediate tergite, $\mathrm{V}$ - ventral, D - dorsal, C - coxa, $\mathrm{Tr}$ - trochanter,

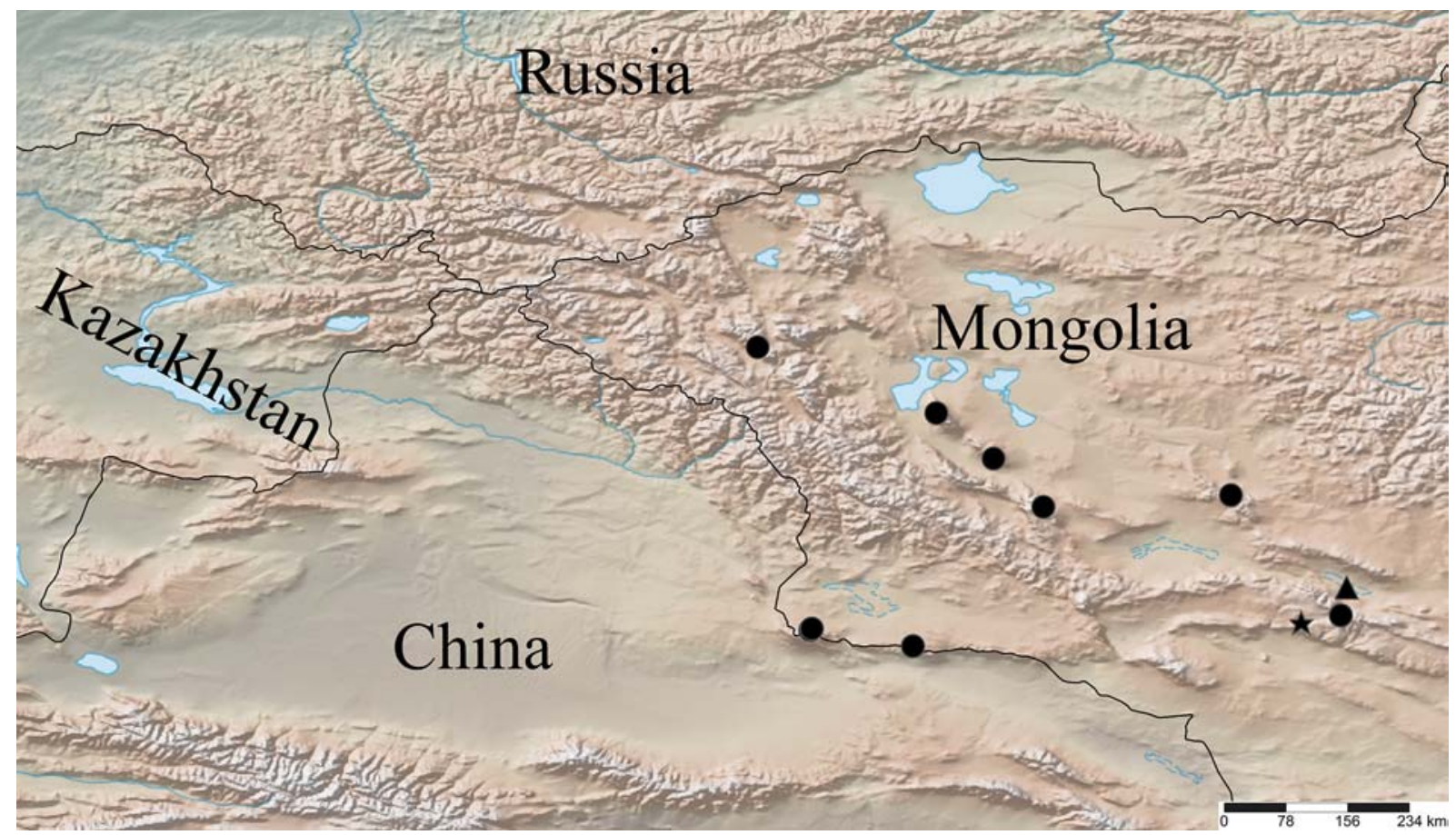

Fig. 1. Distribution of Schizotergitius altajicus Loksa, 1978: the type locality (asterisk), the type locality of S. altajicus f. gracilis Loksa, 1978 (triangle), non-type material (circles).

Рис. 1. Распространение Schizotergitius altajicus Loksa, 1978: типовое местонахождение (звездочка), типовое местонахождение S. altajicus f. gracilis Loksa, 1978 (треугольник), нетиповой материал (круг). 
$\mathrm{P}$ - prefemur, $\mathrm{F}$ - femur, Ti - tibia, Ts 1 - tarsus 1 , Ts 2 - tarsus 2 , Tsi - tarsi, a - anterior, $\mathrm{m}$ - median, $\mathrm{p}$ - posterior.

\section{Taxonomic part}

\section{Schizotergitius Verhoeff, 1930}

Schizotergitius Verhoeff, 1930: 248.

Schizotergitius - Zalesskaja, 1978: 35.

Schizotergitius - Zapparoli, Edgecombe, 2011: 383.

Schizotergitius - Eason, 1986a: 190

Schizotergitius - Farzalieva et al., 2017: 124, table 1

Type species: S. longiventris Verhoeff, 1930.

BRIEF DESCRIPTION. Schizotergitius is characterized by the medium body size $(12-25 \mathrm{~mm})$, the functionally biarticulate tarsi 1-13, 20-segmented antennae, 5-6 ocelli, the small Tömösváry's organ (smaller than the nearest ocellus), the forcipular coxosternite with $2+2$ teeth and porodonts, the absence of posterolateral triangular projections from tergites, the elongated macrotergites (except for TT 2 and 8), TT 3, 5, 8, 10, 12 with posterior median notches, SS elongated, with sutures developed at the posterior margin, the number (2-4) of coxal pores on legs 12-15, the 1-segmented $\sigma^{7}$ gonopods with $2-4$ setae, and the + gonopods with simple claws. See also Key below.

DIAGNOSIS. The genus seems to be especially similar to species of the giganteus-group of the subgenus Ezembius Chamberlin, 1919, genus Lithobius Leach, 1814 [Eason, 1986b], to the genus Disphaerobius Attems, 1926, as well as to species of the genus Hessebius Verhoeff, 1941 with biarticulate tarsi (H. perelae Zalesskaja, 1978, H. plumatus Zalesskaja, 1978, $H$. procurvus Zalesskaja, 1978 and $H$. pervagatus Zalesskaja, 1978) through the functionally biarticulate tarsi of legs 1-13, the antennae composed of 20 antennomeres, Tömösváry's organ smaller or equal in size to the nearest ocellus, the rounded posterior angles of TT, and the 1-segmented $\sigma^{7}$ gonopods. However, Schizotergitius differs well by the elongated macrotergites, as well as the posterior median notches developed on TT 3, 5, 8, 10, 12 in both sexes. See also Key below.

SPECIES COMPOSITION: S. longiventris Verhoeff, 1930 and S. altajicus Loksa, 1978.

REMARK. The $q$ of $S$. longiventris is still unknown.

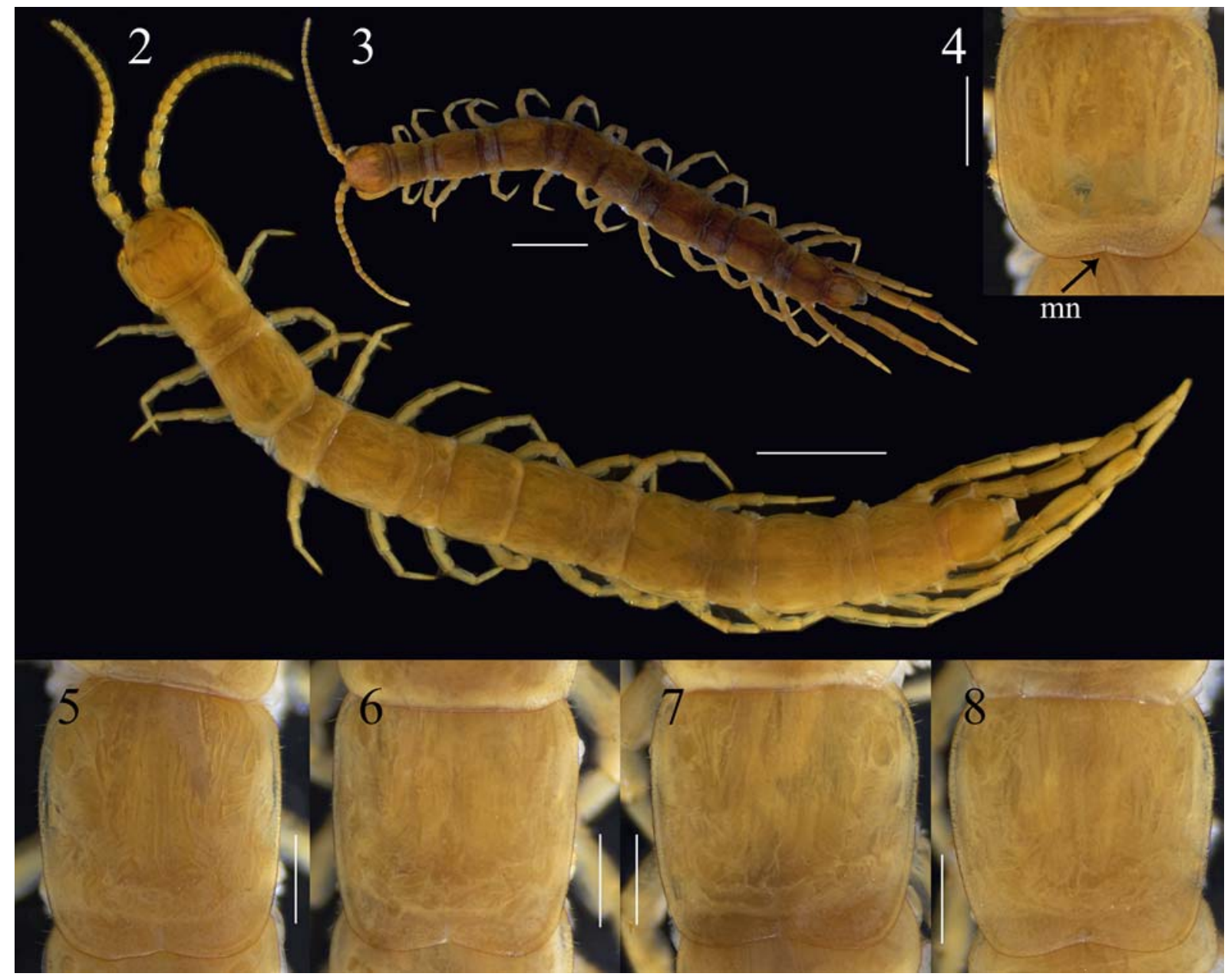

Figs 2-8. Schizotergitius altajicus Loksa, 1978 (2, 4-8 - $0^{7}$ paratypes, $3-\sigma^{7}$ non-type, HNHM chilo-7688), dorsal view: 2, 3 habitus; 4 - tergite $3 ; 5$ - tergite $5 ; 6$ - tergite $8 ; 7$ - tergite $10 ; 8$ - tergite 12 . Abbreviation: $\mathrm{mn}$ - median notch. Scale: $2 \mathrm{~mm}(2,3)$, $0.5 \mathrm{~mm}(4-8)$

Pис. 2-8. Schizotergitius altajicus Loksa, 1978 (2, 4-8 - паратипы $0^{7} \sigma^{7}, 3$ - нетиповой $0^{7}$, HNHM chilo-7688), сверху: 2, 3 общий вид; 4 - тергит $3 ; 5$ - тергит 5; 6 - тергит $8 ; 7$ - тергит 10;8 - тергит 12. Обозначение: $\mathrm{mn}$ - срединная бороздка. Масштаб: 2 мм $(2,3), 0,5$ мм (4-8). 


\section{Schizotergitius altajicus Loksa, 1978}

Figs 1-53.

Schizotergitius altajicus Loksa, 1978: 116-118, figs 10-19. Schizotergitius altajicus forma gracile (sic!) Loksa, 1978: 118. Schizotergitius altajicus - Eason, 1986a: 190.

Schizotergitius altajicus - Dyachkov, 2017: 35.

Schizotergitius - Farzalieva et al., 2017: 124, table 1.

DIAGNOSIS. The species differs from $S$. longiventris by the smaller size (12-21 $\mathrm{mm} v \mathrm{~s} .22 .5-25 \mathrm{~mm}$ in $S$. longiventris), body colouration (yellow or brownish yellow $v s$. a greybrown body with a darker head in $S$. longiventris), the presence of DCa on 15 legs ( $v s$. absent in S. longiventris), as well as the number of dorsal spines on 13P (three dorsal spines vs. a single dorsal spine on $13 \mathrm{P}$ in $S$. longiventris).

BRIEF DESCRIPTION. A medium-sized $(12-21 \mathrm{~mm})$ Lithobiinae with 5-6 ocelli in a single broken row, Tömösváry's organ smaller than the nearest ocelli; usually 20 antennal articles (rarely 17-22); forcipular coxosternite with $2+2$ acute teeth and setiform porodonts; TT 3, 5, 8, 10, 12 with posterior median notches, SS elongated (excluding genital S); legs 1-13 with functionally biarticulate tarsi; legs 15 without accessory spines; DCa present on legs (11)12-15; number of coxal pores, $2-4$ on legs $12-15$; O $15 \mathrm{~F}$ (sometimes 15P) showing more or less evident, indistinct, wide, dorsal and/or dorsolateral sulci, 15Ti with a dorsal or dorsolateral sulcus and a dark blot in distodorsal part covered with numerous setae; $O^{7}$ gonopod 1-segmented with 2-4 setae; + gonopod with $2+2$ gonopodal spurs and a simple, curved and pointed claw, $1^{\text {st }}$ segment without dorsal setae (rarely with 1), $2^{\text {nd }}$ segment with a small, translucent, distodorsal outgrowth shifted mesad (Figs 28-30) and 4-6 dorsal setae, $3^{\text {rd }}$ segment with 2-4 dorsal setae (Figs 31-33, 36-37).

MATERIAL. Type material: paratypes: $12 \sigma^{7} \sigma^{7}, 10$ 오 [vs. 11 $\sigma^{7} \sigma^{7}, 11$ 우, 4 juv. in the original description] (HNHM chilo-7558) (one $\sigma^{7}$ with postpedal segments, forcipules, the left ocellar field, $\mathrm{T} 7$, left $1^{\text {st }}$ and right $14-15^{\text {th }}$ legs missing, probably removed and mounted on slides which could have served for Loksa's [1978] illustrations, but no such slides could be relocated], [Mongolia,

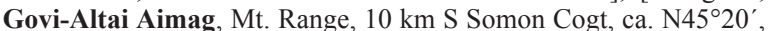
E96 38'] "Gobi Altaj Gebirge, $10 \mathrm{~km} \mathrm{S.} \mathrm{von} \mathrm{Somon} \mathrm{Cogt,} \mathrm{am} \mathrm{Pass,}$ 2500 m, 27.VI.1966 (Nr. 589)"; Schizotergitius altajicus f. gracile Loksa, 1978, types: 3 우 [O holotype, $1 \sigma^{7}$ and $1+$ paratype, according to the original description, but no holotype selected and labeled in the material] (HNHM chilo-7559), [Mongolia, GoviAltai Aimag, between Lake Beger-Nuur and Somon Beger, ca. N45 $44^{\prime}$, E97 $09^{\prime}$ ' "zwischen dem See Beger Nuur und Somon Beger, 1400 m, 25-26.VI.1966 (Nr.579)".

Other material: Mongolia: 4 우 (ASU No. 295), Bayan-Ölgii Aimag, Buratyn-Davaa Pass, N48 $28^{\prime}$, E90 ${ }^{\circ} 27^{\prime}$, mountain stony steppe, $2600 \mathrm{~m}$, 24.VI.2015, coll. AF, OB, DN; 8 O $^{7} 0^{7}$ (ASU No. 320), 24.VI 2015, coll. AF, OB, DN; $1 \sigma^{7}, 1$ (ASU No. 41), Khovd Aimag, Sutai-Uul Mt. Range, Mt Zamlyn-Bogt, scree, N46 $39^{\prime}$ E93 $42^{\prime}, 3810-3860 \mathrm{~m}$, coll. AF; $2 \mathrm{O}^{\top} \mathrm{O}^{\top}, 1$ \% (ASU No. 42), BumbatKhayrhan-Bogt Mt. Range, Mt Degnuul, stony alpine meadow, N47 ${ }^{\circ} 12^{\prime}, \mathrm{E}^{\circ} 03^{\circ} 08^{\prime}, 3000-3200$ m a.s.1., 24.VI.2017, coll. AF; $1 \mathrm{O}^{\mathrm{T}}, 2$ 우 (ASU No. 296), Baitag-Bogd-Uul Mt. Range, Gushoot-Shyneet-

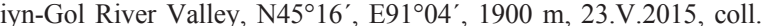

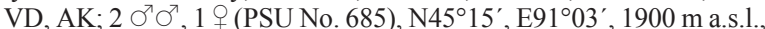
24.V.2015, coll. RY; $2 \sigma^{7} \sigma^{7}$ (ASU No. 293), Dzhargalant-Khairkhan
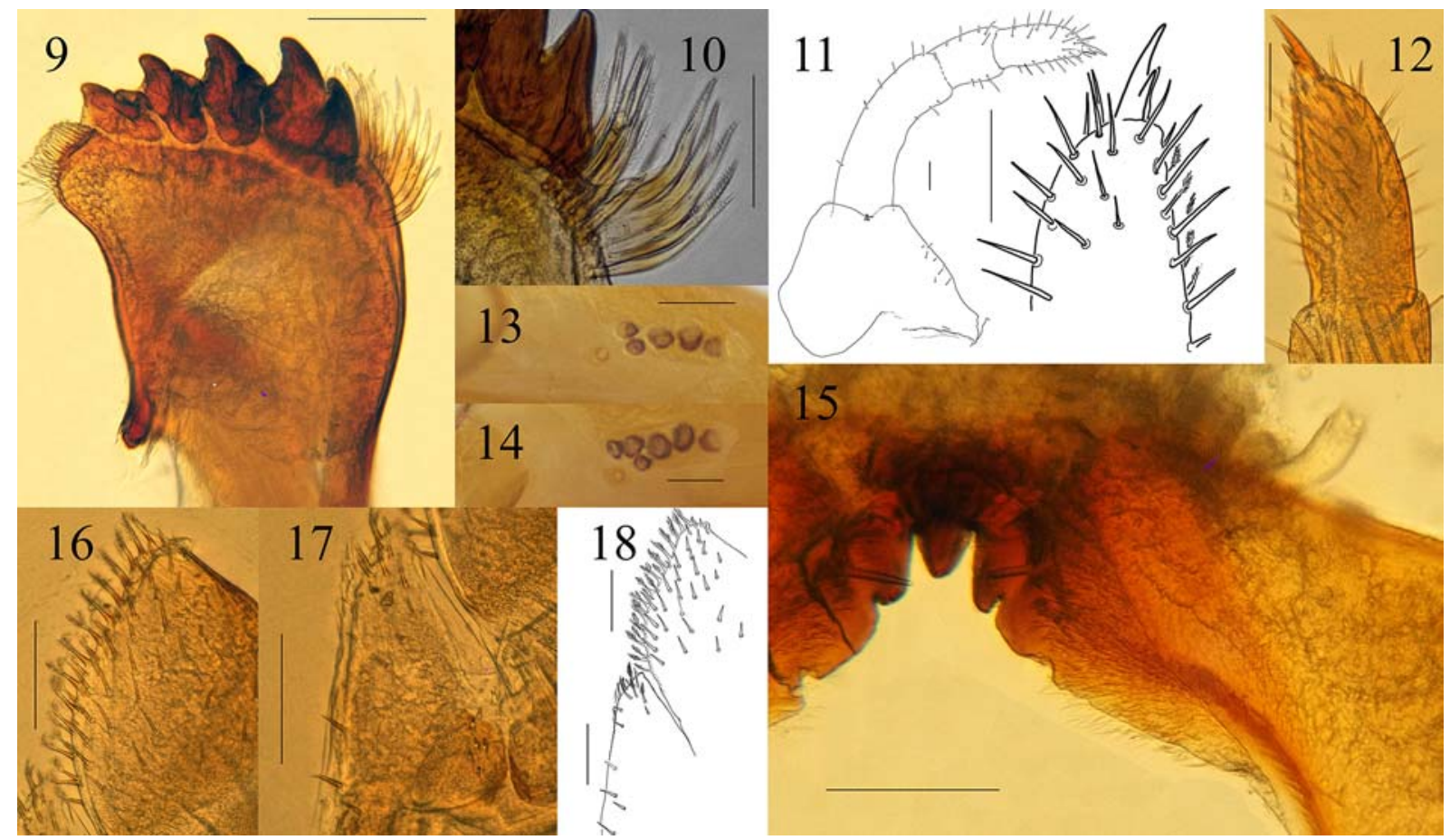

Figs 9-18. Schizotergitius altajicus Loksa, 1978 (O’ paratypes: 13, 14; non-types: 9, 11, 12, 15-18 — PSU No. 668, 10 - ASU No. 295): 9-mandible, anterodorsal view; 10 - aciculae of mandible, anterodorsal view; 11 - right part of second maxillae and its apical part, ventral view; 12 - apical part of second maxillae, dorsal view; 13-14 - ocelli and Tömösváry's organ, lateral view; 15 - central and left parts of labrum, ventral view; 16 - apical part of first maxillae telopodite, ventral view; 17 - coxal projection of first maxillae, ventral view; 18 — terminal article and coxal projection of the first maxillae, ventral view. Scale: $0.1 \mathrm{~mm}(11-12,15-18), 0.5 \mathrm{~mm}(9), 0.05 \mathrm{~mm}(10)$.

Рис. 9-18. Schizotergitius altajicus Loksa, 1978 (паратипы, самцы: 13, 14; нетиповой материал: 9, 11, 12, 15-18 - PSU No. 668, 10 - ASU No. 295): 9 - мандибула, одновременно спереди и сверху; 10 - мандибулярные ацикулы, одновременно спереди и сверху; 11 - правая часть вторых максилл и их апикальная часть, снизу; 12 - вершинная часть вторых максилл, сверху; $13-14-$ глазки и орган Темешвари, сбоку; 15 - центральная и левая части верхней губы, снизу; 16 - вершинная часть телоподита первых максилл, снизу; 17 - коксальный отросток первых максилл, снизу; 18 - вершинная часть телоподита и коксальный отросток первых максилл, снизу. Масштаб: 0,1 мм (11-12, 15-18), 0.5 мм (9), 0,05 мм (10). 

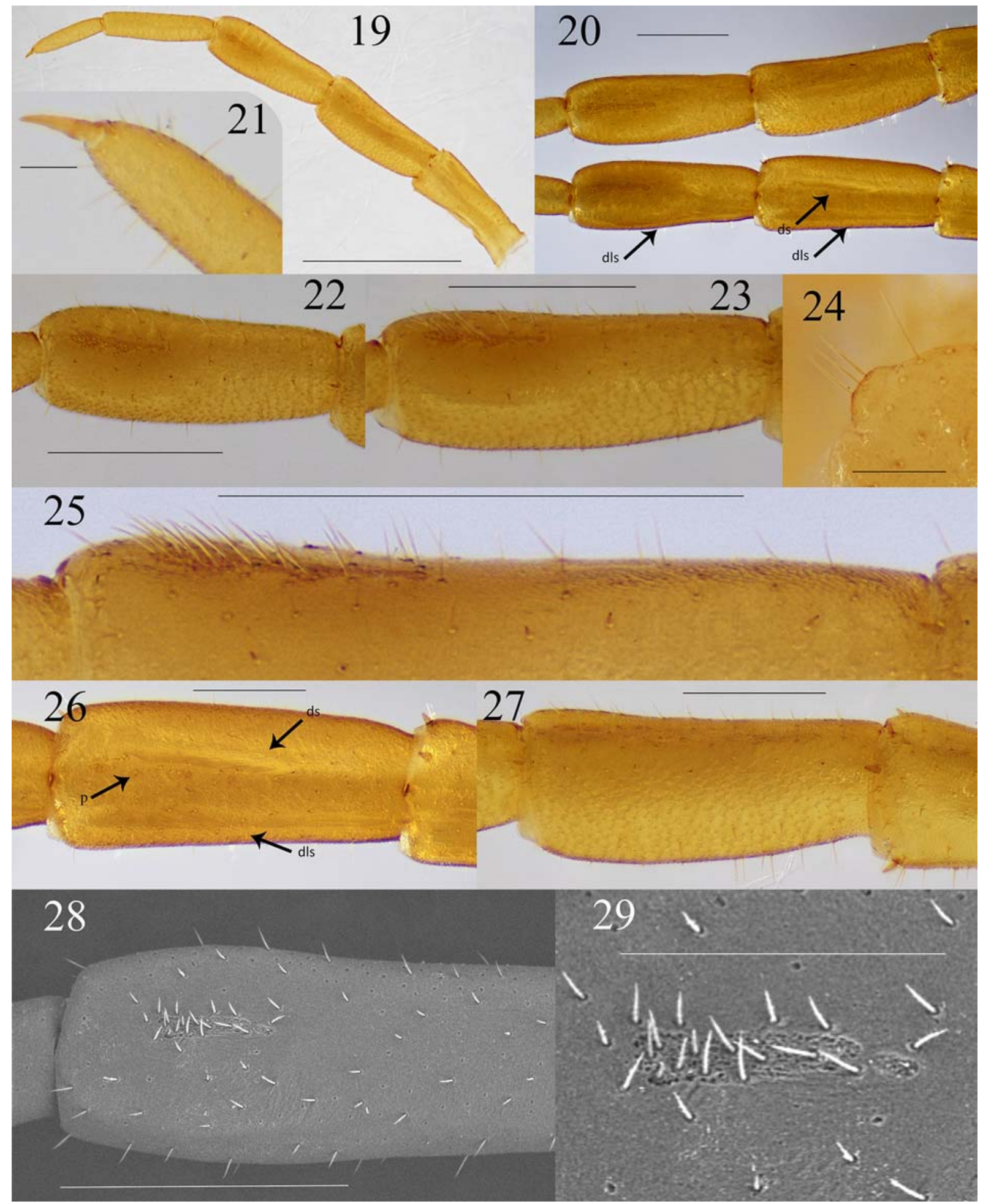

Figs 19-29. Schizotergitius altajicus Loksa, 1978 (male; paratypes: 19-27; nontype: 28, 29 - PSU No. 668): 19 - leg 15, dorsal view; 20 - femur and tibia of legs 15, dorsal view; 21 - claw of leg 15, lateral view; 22 - tibia 15, dorsal view; 23 - same, meso-dorsal view; 24 - gonopod, ventral view; 25 - distal part of tibia 15, mesodorsal view; 26 - femur 15, dorsal view; 27 - same, mesodorsal view; 28 - distal part of tibia 15, dorsal view; 29 - blot on tibia 15, dorsal view. Abbreviations: ds - dorsal sulcus, dls - dorsolateral sulcus, p - pore. Scale: $1 \mathrm{~mm}(19), 0.5 \mathrm{~mm}(20,22,23,25-28), 0.2 \mathrm{~mm}(29), 0.1 \mathrm{~mm}(21,24)$.

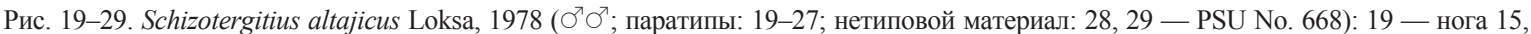
дорсально; 20 - бедро и голень ноги 15 , дорсально; 21 - коготок ноги 15, латерально; 22,23 — голень ноги 15 , соответственно сверху и одновременно изнутри и сверху; 24 — гонопод, вентрально; 25 — дистальная часть голени 15 , мезо-дорсально; 26 — бедро 15 , дорсально; 27 - то же, мезо-дорсально; 28 - дистальная часть голени 15, дорсально; 29 - пятно на голени 15, дорсально. Обозначения: ds дорсальная бороздка, dls - верхнебоковая бороздка, р — пора. Масштаб: 1 мм (19), 0.5 мм (20, 22, 23, 25-28), 0.2 мм (29), 0.1 мм (21, 24). 


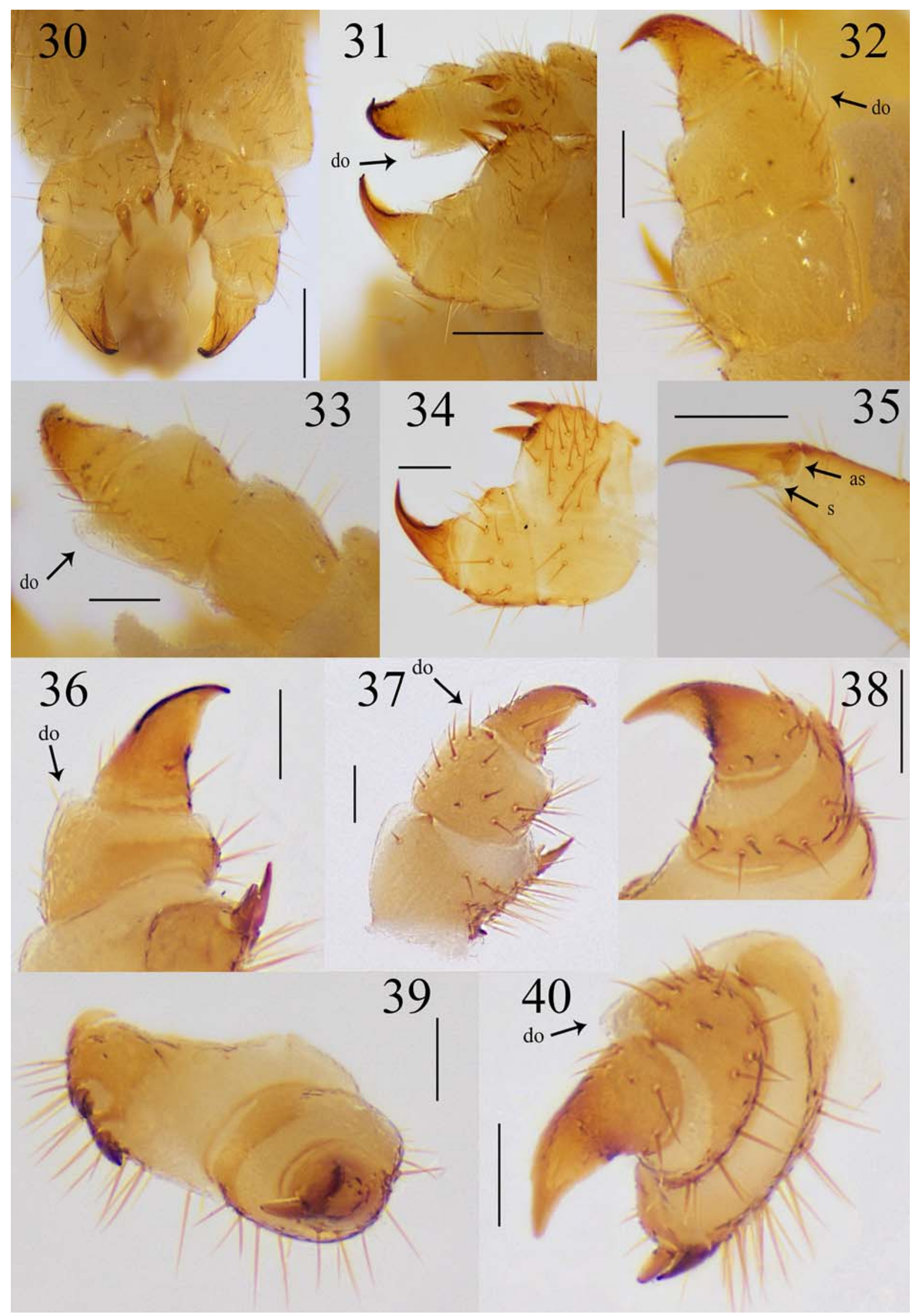


Mt. Range, near source of Ar-Shaatyn-Gol River, mountain stony tundra, N4743', E92 ${ }^{\circ} 9^{\prime}$ ', 3300-3500 m a.s.1., 25.VI.2015, coll. AF $1 \overbrace{}^{7}$ (ASU No. 294), Ikh-Khavtag-Uul Mt. Range, near Zhugentiyn Spring, N45 $04^{\prime}$, E92 $13^{\prime}$, mountain stony steppe with rocks, 2050 $2300 \mathrm{~m}$ a.s.1., 30.VI-2.VII.2015, coll. AF; 1 ○ (PSU No. 668), IkhKhavtag-Uul Mt. Range, near Yargaytiyn-Sayr Ravine, mountain stony steppe with rocks, $\mathrm{N}^{\circ} 5^{\circ} 04^{\prime}$, E92 ${ }^{\circ} 13^{\prime}, 1900-2150 \mathrm{~m}$ a.s.1., 30 31.V.2015, coll. AF; $4 \sigma^{7} \sigma^{7}, 1$ o (HNHM chilo-7686), Govi-Altai Aimag, Cakhir-Khaalgyn-Nuruu Mt. Range, 2-5 km SSE DotiynDavaa Pass, mountain stony tundra with rocks and scree, $\mathrm{N} 45^{\circ} 25^{\prime}$,

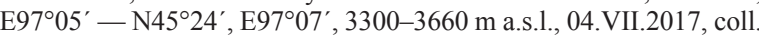
AF; $1 \sigma^{7}$ (HNHM chilo-7688), 04.VII.2017, coll. AF; 1 9 (HNHM chilo-7687), foothill of Cakhir-Khaalgyn-Nuruu Mt. Range, near Mt Dotiyn-Davaa, pass, stony alpine meadow, N45 $25^{\prime}$, E97 $05^{\prime}, 2900$ 3000 m a.s.1., 04-05.VII.2017, coll. AF; $4 \sigma^{7} \sigma^{\top}, 2$ 우 (ZMMU Rc 7892), Khasagtiyn-Nuruu Mt. Range, vicinity of Mt Khoit-BogdUul, mountain stony tundra with rocks and scree, N46 $47^{\prime}, \mathrm{E} 95^{\circ} 50^{\prime}$, 3400-3500 m a.s.1., 08.VII.2017, coll. AF.

REDESCRIPTION. O' paratypes. Body $12-17 \mathrm{~mm}$ long, colouration in alcohol yellow (Fig. 2), cephalic plate slightly brighter; head and body slightly punctate. Head a little broader than T1 (Fig. 41) (ca. 1:0.95).

Antennae with 20 articles ( $1 \sigma^{7}$ with $18+20$ ones, $2 \sigma^{7} \sigma^{7}$ with 19+20), covered with dense, short, dark and erect sensilla. Length to breadth ratio of terminal article, 1:0.4 (Fig. 44). Antennae extending back to ca. T4.

Ocelli: $5-6$ on each side arranged in a single broken row inside a contoured area (Figs 13,14). Tömösváry's organ smaller than nearest ocelli, rounded.

Coxosternum with $2+2$ acute teeth and setiform porodonts, median diastema V-shaped. Shoulders very gently sloping behind porodonts.

Tergites: almost smooth, with short and sparse setae, without posterior triangular projections. TT 3, 5, 8, 10, and 12 elongated (Table 3 ); posterior median notch developed on TT 3, 5, 8, 10 and 12 (caudal part of each of these TT with a well-developed, light, short suture dividing it into two lobes) (Figs 4-8, 51); T15 indistinct. T1 breadth ca. $1.2 \mathrm{~mm}$, T8 breadth ca. $1.45 \mathrm{~mm}$. All microtergites (TT 2, 4, 6, 9, 11, 13) and $\mathrm{T} 7$ with one median and two paramedian, short, translucent sutures at posterior margin (Fig. 50). Caudal margin of Tim slightly sinuate, length to breadth ratio, 1:0.94 (Fig. 42). Caudal part of $14-15^{\text {th }}$ sternites and coxae densely covered with short setae (Fig. 45).

Sternites elongate (except for genital S); posterior margin of SS 1-14 with short sutures (best expressed on SS 2-12).

All Tsi functionally biarticulate, with Ts 1 about 2 times longer than Ts2. Tsi 1-13 each with a ventral row of dark setae especially well-developed on midbody legs. Legs 1-14 each with a posterior accessory spine, legs 1-13 each with a large, pretarsal, anteroventral spine (Fig. 35). Legs 15 without accessory spines (Fig. 21), but with a very small anteroventral spine (interpreted as an accessory spine in the original description). $\mathrm{P}, \mathrm{F}, \mathrm{Ti}$ of legs 15 slightly incrassate and flattened dorsally (Figs 19-20). Legs 14 without visible secondary sexual characters.

$15 \mathrm{~F}$ covered with sparse minute setae and several small, oval, dark pores (Figs 26, 27). Distodorsal part of $15 \mathrm{Ti}$ flattened and with a long, median, dark blot formed by numerous small pores, as well as with numerous dense setae located on and around the dark blot (Figs 22, 23, 25); 15F and $15 \mathrm{Ti}$ with more or less evident, shallow, wide, indistinct dorsal and/or dorsolateral sulci (Figs 20, 26). Leg plectrotaxy as in Table 1.

DCa developed starting with legs (11)12. Coxal pores small, rounded, separated from one another by a distance about 2-4 times greater than their own diameter, formula 3,2(3),2(3),2(3) (Fig. 45). Gonopod (Fig. 24) 1-segmented, with 3-4 very thin and light setae placed at gonopodal middle.

$\sigma^{7}$ variations (non-type material).

Most characteristics as in paratypes, except as follows. Body length 13-21 mm, colouration in alcohol always brownish yellow (Fig. 3). Antennae with 20-22 segments

Table 1. Plectrotaxy of Schizotergitius altajicus Loksa, 1978 (paratypes). Brackets indicate variable spines.

Таблица 1. Распределение шипов на ногах у Schizotergitius altajicus Loksa, 1978 (паратипы). Скобки обозначают вариабельные шипы.

\begin{tabular}{|c|c|c|c|c|c|c|c|c|c|c|c|c|}
\hline \multicolumn{7}{|c|}{$\mathrm{D}$} & \multicolumn{6}{|c|}{$\mathrm{V}$} \\
\hline Legs & $\mathrm{C}$ & $\mathrm{Tr}$ & $\mathrm{P}$ & $\mathrm{F}$ & $\mathrm{Ti}$ & $\mathrm{Ta}$ & Legs & $\mathrm{Tr}$ & $\mathrm{P}$ & $\mathrm{F}$ & $\mathrm{Ti}$ & $\mathrm{Ta}$ \\
\hline 1 & & & ap & $a p$ & $a(p)$ & & 1 & & $p$ & amp & am & \\
\hline $2-9$ & & & ap & ap & ap & & $2-9$ & & $\mathrm{p}$ & amp & am & \\
\hline 10 & & & $a(m) p$ & ap & ap & & 10 & & $(\mathrm{~m}) \mathrm{p}$ & amp & $\mathrm{am}$ & \\
\hline 11 & (a) & & amp & ap & ap & & 11 & & $(\mathrm{~m}) \mathrm{p}$ & amp & $\mathrm{am}$ & \\
\hline 12 & (a) & & amp & (a)p & (a)p & & 12 & & $(\mathrm{~m}) \mathrm{p}$ & amp & $\operatorname{am}(p)$ & \\
\hline 13 & $\mathrm{a}$ & & amp & $\mathrm{p}$ & $\mathrm{p}$ & & 13 & & $(\mathrm{~m}) \mathrm{p}$ & $\mathrm{amp}$ & $\mathrm{am}$ & \\
\hline 14 & $a$ & & amp & $p$ & (p) & & 14 & $\mathrm{~m}$ & (a) $\mathrm{mp}$ & $\operatorname{am}(p)$ & $\mathrm{a}(\mathrm{m})$ & \\
\hline 15 & $a$ & & amp & $\mathrm{p}$ & & & 15 & $\mathrm{~m}$ & amp & $\mathrm{am}$ & & \\
\hline
\end{tabular}

Figs 30-40. Schizotergitius altajicus Loksa, 1978 (†ㅇ paratypes: 30-33, 35; ㅇ non-type: 34, 36-40 - ASU No. 295): $30,31-$ gonopods, ventral and ventrolateral views, respectively; 32 - right gonopod, dorsolateral view; 33, 34 — left gonopod, dorsal and lateral views, respectively; 35 - pretarsus of leg 14; 36 - fragment of right gonopod, ventrolateral view; 37 - left gonopod, dorsolateral view; 38 - right gonopod segments 2 and 3, lateral view; 39, 40 - right gonopod, distal and distodorsal views, respectively. Abbreviations: as — posterior accessory spine; do - distodorsal outgrowth, $\mathrm{s}$ - anteroventral spine. Scale: $0.2 \mathrm{~mm}(30,31), 0.1 \mathrm{~mm}(32-40)$.

Рис. 30-40. Schizotergitius altajicus Loksa, 1978 (паратипы 우: 30-33, 35; нетиповая +: 34, 36-40- ASU No. 295): 30, $31-$ гоноподы, соответственно снизу и одновременно снизу и сбоку; 32 - правый гонопод, одновременно сверху и сбоку; 33,34 - левый гонопод, соответственно сверху и сбоку; 35 - коготь ноги 14; 36 - фрагмент правого гонопода, одновременно снизу и сбоку; 37 — левый гонопод, одновременно сверху и сбоку; 38 - сегменты 2 и 3 правого гонопода, сбоку; 39,40 - правый гонопод дистально и дистодорсально. Обозначения: as - дополнительный коготок; do - дистальный верхний вырост, s - передне-нижняя щетинка. Масштаб: 0,2 мм (30, 31), 0,1 мм (32-40). 
$(19+20$ in 1 specimen), terminal segment of antennae as in Fig. 43.

Dental margin of forcipular coxosternite as in Fig. 47.

Sides of labrum with well-expressed fringes of numerous thin bristles; a pair of setae projecting across labral midpiece (Fig. 15). Gnathal edge of mandible with numerous pulvilli, 4 pairs of well-developed teeth and 10-12 rather thick and serrate aciculae (Figs 9-10). First maxillae: edge with more than 20 plumose bristles and simple setae as well (Figs 16-
18). Second maxillary telopodite (Figs 11,12) with bristles, both simple and plumose, at tip (more than 25 plumose bristles on internal side).

Hind margin of Tim strongly sinuate, and posterior margins of lobes slightly deflexed down.

$15 \mathrm{P}$ rarely with indistinct dorsal and/or dorsolateral sulci; 15 F dorsally with $3-7$ oval pores (Figs 48,49 ). Dark blot on $15 \mathrm{Ti}$ consisting of numerous, densely located, small pores (Figs 28, 29). Leg plectrotaxy as in Table 2.

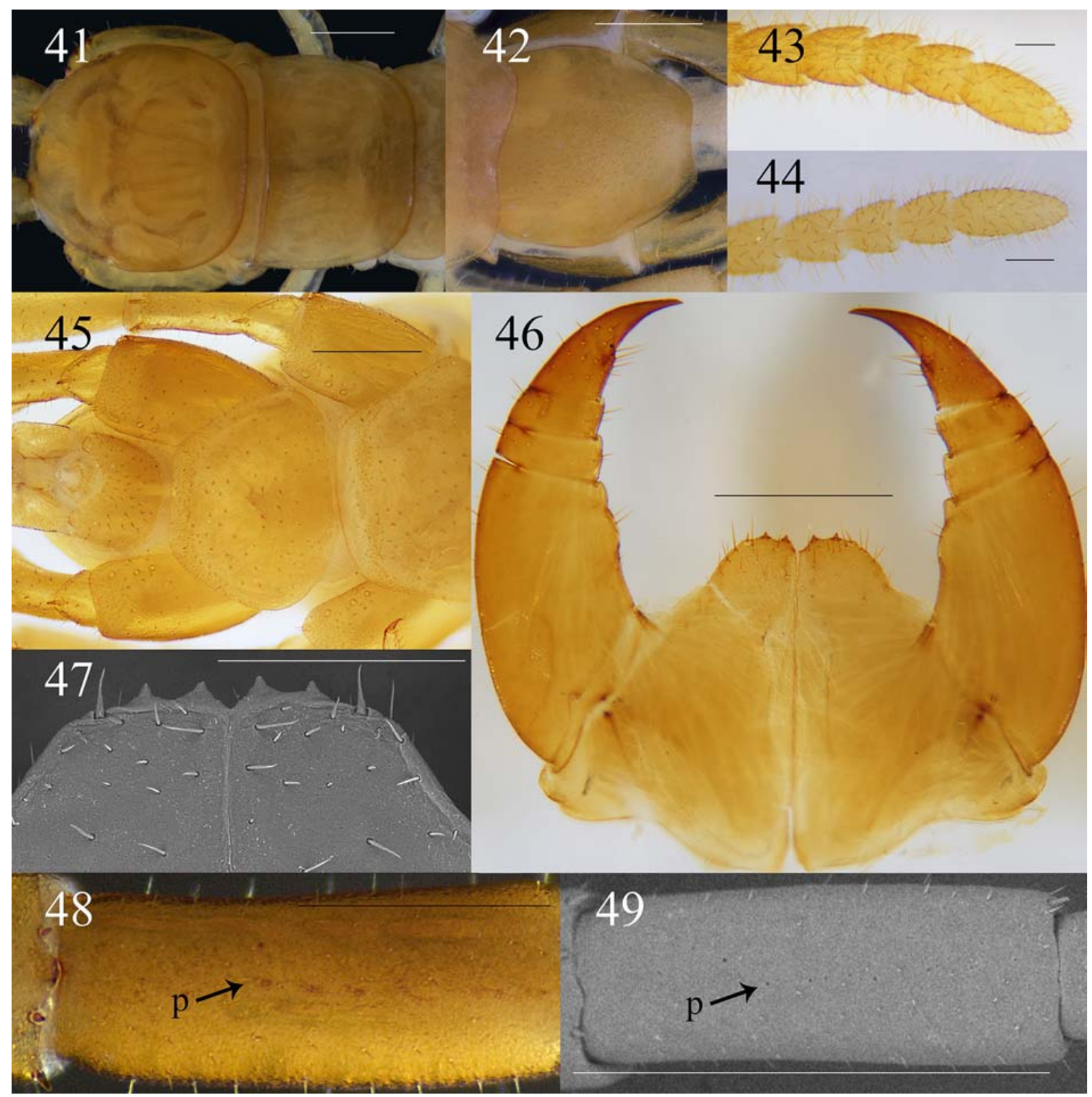

Figs 41-49. Schizotergitius altajicus Loksa, 1978 (paratypes: 41, 42, 44, 45; non-types: 43, 46, 48 — ASU No. 295, 47, 49 — PSU No. 668): 41 - front body fragment of $\sigma^{7}$, dorsal view; 42 - intermediate tergite of $\sigma^{7}$, dorsal view; 43, 44 - fragment of $\sigma^{7}$ antenna, lateral and dorsal views, respectively; 45 - rear body fragment of $O^{7}$, ventral view; $46-$ forcipule, ventral view; 47 - dental margin of $O^{7}$ forcipular coxosternite, ventral view; $48,49-\mathrm{O}^{7}$ femur 15, dorsal view. Abbreviation: $\mathrm{p}$ - pore. Scale: $1 \mathrm{~mm}(49), 0.5 \mathrm{~mm}(41,42,45$,

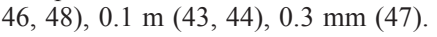

Рис. 41-49. Schizotergitius altajicus Loksa, 1978 (паратипы: 41, 42, 44, 45; нетиповой материал: 43, 46, 48 - ASU No. 295, 47, 49 - PSU No. 668): 41 - передняя часть тела $0^{7}$, сверху; 42 - промежуточный тергит $\bigcirc^{7}$, сверху; 43, 44 - фрагмент антенны $0^{7}$, соответственно сбоку и сверху; 45 - задняя часть тела $\bigcirc^{7}$, снизу; 46 - ногочелюсть 9 , снизу; 47 - зубной край коксостернита ногочелюсти О ${ }^{7}$, снизу; 48, 49 - бедро 15 у $0^{7}$, сверху. Обозначение: $\mathrm{p}$ - пора. Масштаб: 1 мм (49), 0,5 мм $(41,42,45,46,48), 0,1$ мм $(43,44), 0,3$ мм (47). 
SS 14 and 15, coxae 13-15 (Fig. 45), as well as ventral sides of $\mathrm{P}, \mathrm{F}$ and Ti 12 and 13 covered with very small and dense setae in rear parts and, in some cases, even all along the articles (Fig. 54). Genital sternite short and broad, covered with long setae.

Each gonopod with 3 (rarely 2) setae.

o paratypes. Most characteristics as in $\sigma^{7}$ paratypes, but body $(11-16 \mathrm{~mm})$ and TT shorter than in $\sigma^{7}$ (Table 3$)$. T1 breadth ca. $1.01 \mathrm{~mm}$, T8 breadth ca. $1.29 \mathrm{~mm}$. Coxosternum usually with $2+2$ acute teeth $(1$ specimen with $2+1$, another one with $2+3$ ones). Formula of coxal pores, 3,3,3,3.
Gonopod with $2+2$ acute spurs (Figs 30,31) and a simple, pointed and curved claw; $1^{\text {st }}$ segment without dorsal setae, but with 1-3 short and 3 longer medial setae, $2^{\text {nd }}$ segment with a small, translucent, distodorsal outgrowth shifted mesad and 4-6 dorsal setae (vs. 7 in the holotype in the original description, but, according to the illustrations [Loksa, 1978: figs 17, $19]$, a lateral seta similar in appearance was also counted), $3^{\text {rd }}$ segment with 2-3 dorsal setae (Figs 32, 33).

o variations (non-type material).

Body $12-20 \mathrm{~mm}$ long, antennae usually with 20 antennomeres $(1+$ with $17+19)$.

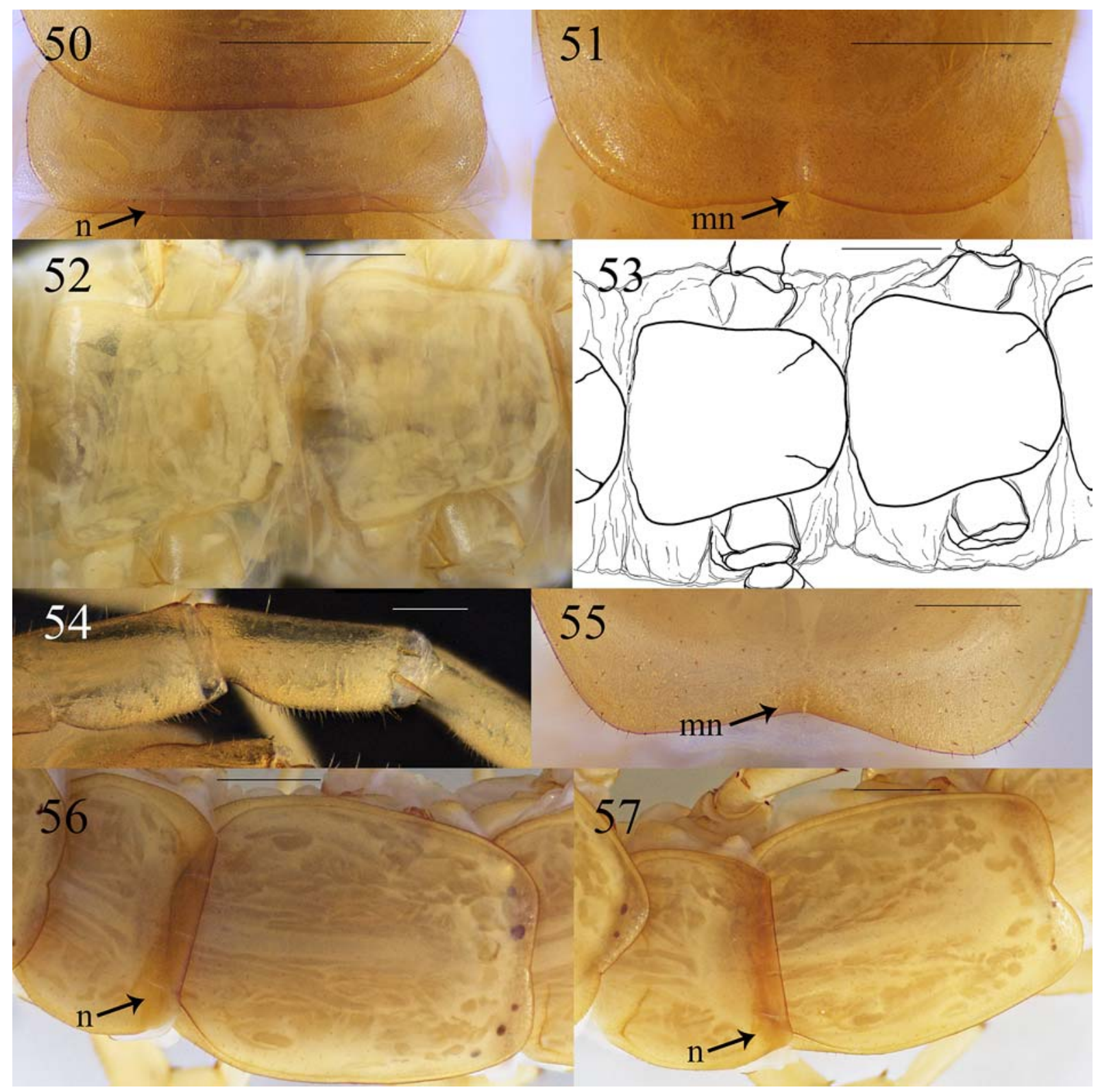

Figs 50-57. Schizotergitius altajicus Loksa, 1978 (50-54, paratypes) and Lithobius giganteus Sseliwanoff, 1881 (55-57, ○', ASU No. 318; Mongolia, Khovd Aimag; dorsal view): 50 — O tergite 2, dorsal view; 51 - rear part of $\mathrm{O}^{7}$ tergite 5, dorsal view; 52,53 - 9 sternites 7 and 8 , ventral view; 54 - prefemur and femur of $\mathrm{O}^{7}$ leg 13, ventrolateral view; 55 - rear part of tergite $12 ; 56$ — tergites 4 and $5 ; 57$ tergites 9 and 10. Abbreviations: $\mathrm{n}$ - notch, $\mathrm{mn}$ - median notch. Scale: $1 \mathrm{~mm}(56,57), 0.5 \mathrm{~mm}(50-53,55), 0.2 \mathrm{~mm}(54)$.

Рис. 50-57. Schizotergitius altajicus Loksa, 1978 (50-54, паратипы) и Lithobius giganteus Sseliwanoff, 1881 (55-57, O’7, ASU No. 318; Монголия, Ховд Аймак; сверху): 50 — тергит 2 у $0^{7}$, сверху; 51 — задняя часть тергита 5 у $0^{7}$, сверху; 52,53 - стерниты 7 и 8 у 9 , снизу; 54 - предбедро и бедро 13 -й пары у $0^{7}$, одновременно снизу и сбоку; 55 - задняя часть тергита $12 ; 56$ — тергиты 4 и $5 ; 57$ — тергиты 9 и 10. Обозначения: $\mathrm{n}$ - бороздка, $\mathrm{mn}$ - срединная бороздка. Масштаб: 1 мм $(56,57), 0,5$ мм $(50-53,55), 0,2$ мм (54). 
Table 2. Plectrotaxy of Schizotergitius altajicus Loksa, 1978 (non-types). Brackets indicate variable spines. Таблица 2. Распределение шипов на ногах у Schizotergitius altajicus Loksa, 1978 (нетиповой материал).

Скобки обозначают вариабельные шипы.

\begin{tabular}{|c|c|c|c|c|c|c|c|c|c|c|c|c|}
\hline \multicolumn{7}{|c|}{$\mathrm{D}$} & \multicolumn{6}{|c|}{$\mathrm{V}$} \\
\hline Legs & $\mathrm{C}$ & $\mathrm{Tr}$ & $\mathbf{P}$ & $\mathrm{F}$ & $\mathrm{Ti}$ & $\mathrm{Ta}$ & Legs & $\mathrm{Tr}$ & $\mathrm{P}$ & $\mathrm{F}$ & $\mathrm{Ti}$ & $\mathrm{Ta}$ \\
\hline 1 & & & $(a)(p)$ & ap & $\mathrm{a}$ & & 1 & & $\mathrm{p}$ & amp & $\mathrm{am}$ & \\
\hline $2-7$ & & & (a)p & ap & ap & & $2-7$ & & $\mathrm{p}$ & amp & $\mathrm{am}$ & \\
\hline 8 & & & (a)(m)p & $a p$ & ap & & 8 & & $p$ & amp & $\mathrm{am}$ & \\
\hline 9 & & & (a)(m)p & ap & ap & & 9 & & (a)(m)p & amp & $\mathrm{am}$ & \\
\hline $10-11$ & & & $a(m) p$ & ap & ap & & $10-11$ & & (a)(m)p & amp & am & \\
\hline 12 & (a) & & (a)mp & $\mathrm{p}$ & $\mathrm{p}$ & & 12 & & (a)(m)p & amp & am & \\
\hline 13 & $\mathrm{a}$ & & (a)(m)p & $p$ & (a)p & & 13 & & $(\mathrm{a})(\mathrm{m}) \mathrm{p}$ & amp & am & \\
\hline 14 & $a$ & & (a)mp & $\mathrm{p}$ & (p) & & 14 & $\mathrm{~m}$ & amp & (a)m(p) & am & \\
\hline 15 & $\mathrm{a}$ & & $a(m) p$ & (p) & (p) & & 15 & $\mathrm{~m}$ & $\mathrm{a}(\mathrm{m})(\mathrm{p})$ & (a)m & (a) & \\
\hline
\end{tabular}

Table 3. Average lengths and breadths of tergites of $0^{7} \sigma^{7}$ and $O P$ in Schizotergitius altajicus Loksa, 1978

(paratypes)

Таблица 3. Средние значения длины и ширины тергитов у самок и самцов Schizotergitius altajicus Loksa, 1978

(паратипы).

\begin{tabular}{|c|c|c|c|c|}
\hline \multirow{3}{*}{$\begin{array}{l}\text { No. of } \\
\text { tergite }\end{array}$} & \multicolumn{2}{|c|}{ Male } & \multicolumn{2}{|c|}{ Female } \\
\hline & \multicolumn{4}{|c|}{$\mathrm{mm}$, ca. } \\
\hline & length & width & length & width \\
\hline 1 & 0.87 & 1.2 & 0.73 & 1.01 \\
\hline 2 & 0.45 & 1.09 & 0.46 & 0.98 \\
\hline 3 & 1.4 & 1.23 & 1.15 & 1.12 \\
\hline 4 & 0.52 & 1.19 & 0.51 & 1 \\
\hline 5 & 1.51 & 1.34 & 1.27 & 1.2 \\
\hline 6 & 0.6 & 1.32 & 0.62 & 1.2 \\
\hline 7 & 1.21 & 1.43 & 1.09 & 1.26 \\
\hline 8 & 1.51 & 1.45 & 1.23 & 1.3 \\
\hline 9 & 0.65 & 1.36 & 0.67 & 1.27 \\
\hline 10 & 1.53 & 1.47 & 1.28 & 1.36 \\
\hline 11 & 0.71 & 1.39 & 0.62 & 1.3 \\
\hline 12 & 1.50 & 1.46 & 1.2 & 1.31 \\
\hline 13 & 0.54 & 1.16 & 0.49 & 1.21 \\
\hline 14 & 1.27 & 1.34 & 1.07 & 1.17 \\
\hline 15 & \multicolumn{4}{|c|}{ indistinct } \\
\hline 16 & 1 & 0.94 & 0.87 & 0.76 \\
\hline
\end{tabular}

Forcipular coxosternum as in Fig. 46.

Posterior margin of SS 1-14 with short sutures (Figs 52, 53). Formula of coxal pores, 3(4),3,3,2(4).

Gonopods: each with $2+2$ acute spurs (Fig. 34) and a simple, pointed and curved claw (Figs 36-40) with irregular edges (often broken off); $1^{\text {st }}$ segment mostly without dorsal setae (1 dorsal seta on one or both gonopods presents (Fig. 37 ) in 3 of 4 우 from Bayan-Ölgii Aimag (ASU No. 295)), with up to 6 medial setae, $2^{\text {nd }}$ with $4-6,3^{\text {rd }}$ with $3-4$ dorsal setae in a single row; $2^{\text {nd }}$ segment with a small, distodorsal and transparent outgrowth shifted mesad.
DISTRIBUTION. Western Mongolia: Bayan-Ölgii, Khovd and Govi-Altai Aimags [Loksa, 1978; Dyachkov, 2017], summarized in Fig. 1.

REMARKS. Three specimens of S. altajicus f. gracile (recte: gracilis) are labeled as $1 \sigma^{7}$ and 2 + + in the original description [ $O$ holotype, $1 \sigma^{7}$ and $1+$ paratype], but actually all three are + + $:$ one + has postpedal segments damaged, and legs 15 are devoid of the secondary sexual characters of the $\sigma^{7}$. No holotype is labeled as such in the vial.

An important feature of $S$. altajicus is its remarkable sexual dimorphism. $\sigma^{7} \sigma^{7}$ show the body and macrotergites more strongly elongated than $+\bigcirc \mathrm{do}$, but this character is not unique, as the same pattern is observed in the Asiatic Lithobius giganteus Sseliwanoff, 1881 [Eason, 1986a]. This similarity between $S$. altajicus and L. giganteus was noted by Eason [1986a] who mentioned tergal modifications in some specimens of L. giganteus (Figs 55-57), as well as "tergite 12 in the fragmented female of [Lithobius] mongolicus [now $L$. giganteus] is exactly the same as that of tergite 5 in $S$. altajicus". However, S. altajicus differs from L. giganteus in the shorter body, as well as the presence of the well-developed secondary sexual modifications of $\sigma^{7}$ legs 15 : $15 \mathrm{~F}$ has a row of 3-7 oval and dark pores, $15 \mathrm{Ti}$ shows a dark, distodorsal, elongate blot (consisting of numerous pores) covered with dense and short setae. Similarly dense setae are also present in another Asiatic genus, Disphaerobius Attems, 1926, but these are located on a rounded outgrowth of $\mathrm{Ti}$, while a blot is absent [Farzalieva et al., 2017: fig. 5].

The following eight genera of Lithobiidae are presently known to occur in Central Asia: Bothropolys Wood, 1862 (Ethopolyinae Chamberlin, 1915), Australobius Chamberlin, 1920, Disphaerobius Attems, 1926, Hessebius Verhoeff, 1941, Lithobius Leach, 1814, Schizotergitius Verhoeff, 1930, and Validifemur Ma, Song \& Zhu, 2007 (Lithobiinae Verhoeff, 1907) [Loksa, 1978; Zalesskaja, 1978; Eason, 1997; Ma et al., 2007, 2014; Farzalieva et al., 2017; Ganske et al., 2020]. Now that the identity of Schizotergitius has become clarified, we can propose the following key for their separation.

A KEY TO THE GENERA OF LITHOBIIDAE REPORTED FROM Central Asia

1 Coxal pores scattered ................................ Bothropolys - Coxal pores arranged in a single row (Fig. 45) ............ 2

2 Tömösváry's organ located below interior row of ocelli Australobius

— Tömösváry's organ located level with interior row of ocelli (Figs 13, 14) .................................................. 3 
3 Tergites 3, 5, 8, 10, 12 with posterior median notches (Figs $4-8,51) \ldots$ Schizotergitius

- Tergites without median notches

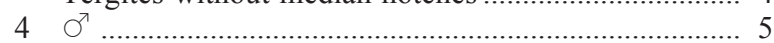

$-9$

5 Tergites 12-14 (or some of them) modified: serrate and sometimes broadened .......................... Disphaerobius

- Tergites neither serrate nor broadened

6 Leg-pair 15 without falciform spines Hessebius and Lithobius

- Leg-pair 15 with strong falciform spines ... Validifemur

7 Second 9 gonopodal article with neither a distodorsal protuberance nor an outgrowth

... 8

- Second 9 gonopodal article with a distodorsal protuberance or outgrowth

9

8 Tarsus of legs 1-13 without any marking on dorsal side, but with a trace of bipartite division on ventral side .....

Validifemur

- Tarsus of legs 1-13 with or without a bipartite division

Lithobius

9 Second $q$ gonopodal article with a more or less developed protuberance, often with setae or spines ....... Hessebius

- Second ${ }_{+}$gonopodal article without such protuberance, but with a small, oval, distodorsal outgrowth directed mesad, always with neither setae nor spines Disphaerobius

Acknowledgements. We are most grateful to S.I. Golovatch (Moscow) who kindly checked the English of an advanced draft. We are highly obliged to A.A. Fomichev who donated us his material for study. The first author is grateful to R.V. Yakovlev who organized his trip to Budapest, and to O.V. Fast, L.A. Khvorova, and A.V. Vaganov (all Barnaul, Russia) who kindly provided FAST software for taking the measurements.

Competing interests. The authors declare no competing interests.

\section{References}

Bonato L., Edgecombe G.D., Lewis J.G.E., Minelli A., Pereira L.A., Shelley R.M., Zapparoli M. 2010. A common terminology for the external anatomy of centipedes (Chilopoda) // ZooKeys. Vol.69. P.17-51. https://doi.org/10.3897/zookeys.69.737

Dyachkov Yu.V. 2017. New records of lithobiid centipedes (Chilopoda: Lithobiomorpha) from western Mongolia // Far Eastern Entomologist. No.345. P.34-36.

Eason E.H. 1986a. On the synonymy of Lithobius giganteus Sseliwanoff, 1881 and the taxonomic status of Porobius Attems,
1926 (Chilopoda) // Annalen des Naturhistorischen Museums in Wien. Vol. 87. P.181-192.

Eason E.H. 1986b. The Lithobiidae of Afghanistan with descriptions of three new species of the giganteus-group of Lithobius subgenus Ezembius, and a key to the central Asiatic species of this group (Chilopoda, Lithobiomorpha) // Steenstrupia. Vol.12. No.2. P.49-60.

Eason E.H. 1997. On some Lithobiomorpha from the mountains of Kizghizia and Kazakhstan (Chilopoda) // Arthropoda Selecta. Vol.6. No.1/2. P.117-121.

Edgecombe G.D. 2004. Monophyly of Lithobiomorpha (Chilopoda): New characters from the pretarsal claws // Insect Systematics \& Evolution. Vol.35. P.29-41.

Farzalieva G.Sh., Nefediev P.S., Tuf I.H. 2017. Revision of Disphaerobius Attems, 1926 (Chilopoda: Lithobiomorpha: Lithobiidae: Pterygoterginae), a centipede genus with remarkable sexual dimorphism // Zootaxa. Vol.4258. No.2. P.121-137.

Ganske A-S., Vahtera V., Dányi L., Edgecombe G.D., Akkari N. 2020. Phylogeny of Lithobiidae Newport, 1844, with emphasis on the megadiverse genus Lithobius Leach, 1814 (Myriapoda, Chilopoda) // Cladistics. Vol.37. P.162-184.

Krasheninnikov A.B. 2011. Mounting technique of entomological preparations in sandarac medium // Euroasian Entomological Journal. Vol.10. No.3. P.278-279.

Loksa I. 1978. Chilopoden aus der Mongolei (Arthropoda: Tracheata, Chilopoda) // Annales Historico-Naturales Musei Nationalis Hungarici. T.70. S.111-120.

Ma H.-Q., Song D.-X., Zhu M.-S. 2007. A new genus and two new species of lithobiid centipedes (Chilopoda: Lithobiomorpha) from China // Zootaxa. Vol.1460. P.25-34.

Ma H.-Q., Pei S., Hou X., Zhu T., Wu D., Gai Y. 2014. An annotated checklist of Lithobiomorpha of China // Zootaxa. Vol.3847. No.3. P.333-358.

Pei S., Ma H.-Q., Zapparoli M., Zhu M. 2010. A review of the Chinese species of Hessebius Verhoeff, 1941 (Chilopoda: Lithobiomorpha: Lithobiidae) // Zootaxa. Vol.2631. P.51-61.

Shorthouse D.P. 2010. SimpleMappr, an online tool to produce publication-quality point maps, accessed at http://www. simplemappr.net on 22-III-2021.

Vaganov A.V., Fast O.V., Khvorova L.A. 2020. [Development of a software module for analysis of images of biological micro- and macro-objects] // MAK: Matematiki - Altaiskomu Krayu. Vol.2. P.154-156 [in Russian].

Verhoeff K.W. 1930. Über Myriapoden aus Turkestan // Zoologischer Anzeiger. Bd.91. H.9/12. S.223-226.

Zalesskaja N.T. 1978. [Identification book of the lithobiomorph centipedes of the USSR (Chilopoda, Lithobiomorpha)]. Moscow: Nauka. 212 pp. [In Russian]

Zapparoli M., Edgecombe G.D. 2011. [Order Lithobiomorpha] // Minelli A. (Ed.). Treatise on Zoology - Anatomy, Taxonomy, Biology. The Myriapoda the Myriapoda. Vol. 1. Leiden-Boston: Brill. P.371-389. 